\title{
Big Data in Gerontology
}

\author{
Christopher Steven Marcum, Ph.D. \\ National Institutes of Health \\ Bethesda, MD USA \\ chris.marcum@nih.gov
}

Note: This invited 500-1000 word entry is under consideration for inclusion in the Springer Encyclopedia of Gerontology and Population Aging co-edited by Danan Gu and Mathew E. Dupre (editors-in-chief) and Kenneth Land and Anthony Bardo (Methods in Gerontology section editors).

\section{Definition}

There are many definitions of "Big Data" as virtually every scientific research enterprise adapts to the emergence of the phenomenon. In gerontology, we might consider Big Data to be defined as any set of observations that exceeds the capacities of traditional gerontological approaches to store, analyze, or make inferences due to its volume, velocity, or complexity. Thus, Big Data is not simply a large number of observations in a dataset but also can be defined in terms of how such a dataset is handled; including, possibly, whether it has lasting clinical, policy, or research significance (Lynch, 2008).

\section{Overview}

Under this definition, Big Data has long been of importance in gerontology. Indeed, many existing datasets in the field have Big Data characteristics. In addition to the traditional repeated measures and cross-sectional observations on individuals, the Health and Retirement Study (HRS), for example, also collects a host of biomarker and genomic data (Weir, 2017). Such additions add both complexity and volume to the project. Complexity results from the nesting of analytes and genotypes within samples, which are in-turn nested within individuals and may also be linked to bibliographical annotations and phenotypes. Increased volume comes from the fact that genetic and genomic data require vast storage capacity and efficient retrieval algorithms (Singer, 2011).

Of course, biomarker collections attached to aging studies are only one such example of Big Data and there are many others of great gerontological interest (see the set curated by the Minnesota Population Center, for instance, via Sobek et al., 2011). One such specific example of an existing study consisting of Big Data is the American Time Use Survey (ATUS). With its many years of repeated cross-sections of a large representative sample of American adults' daily activity diaries linked to household-, activity-, and individual-level data, it is one of the most complex data products on human behavior available to the public (Ovadia, 2013). In addition to their complexity and size, the HRS and ATUS are characteristic of 
Big Data due to their lasting impact on the field of gerontology with thousands of publications based on research of each study.

Big Data is often at the forefront of gerontological research. New innovations in remote health monitoring and sensor technology (Peine \& Nevin, 2018; Schulz et al., 2015), brain and systems biology imaging (Maher et al., 2018), social network collection (Cornwell et al., 2014), and digital trace and online behavior streams (Maddox, 2018) are increasingly becoming available to collect data on older adults. With such an influx of these datasets, there are worries that Big Data and unsupervised analyses will replace theoretical and hypothesis driven research questions. Notably, prior to much influence on life course perspective and biological theories of aging, gerontology was historically labelled as a field that was "data rich and theory poor" (Birren \& Bengtson, 1988).

\section{Future directions for research}

Based on the relatively recent publications listed here, however, this lament has not aged-well as these and other gerontologists have been mindful to deploy theories of aging, health, and social behavior in collecting, analyzing, and interpreting results from these novel datasets. A more relevant issue to the field associated with Big Data, however, is that of research participant privacy. How to protect older adult's privacy and anonymity, and how to clearly articulate the risks of participation, in a context where multiple sources of data could be combined to uniquely identify participants should be a primary concern for gerontologists employing Big Data in their own research as many older adults share concerns of privacy in the collection and sharing of Big Data (Boise et al., 2013). Fortunately, researchers and ethicists are actively addressing these concerns and we can remain cautiously optimistic about the role of Big Data in revealing new discovery in health and welfare of older people while protecting them from breaches of privacy.

\section{References}

Birren J, Bengtson V. (1988). Preface, in Emergent theories of aging. New York: Springer. ix--x

Boise, L., Wild, K., Mattek, N., Ruhl, M., Dodge, H. H., \& Kaye, J. (2013). Willingness of older adults to share data and privacy concerns after exposure to unobtrusive in-home monitoring. Gerontechnology: international journal on the fundamental aspects of technology to serve the ageing society, 11(3), 428--435.

Cornwell, B., Marcum, C., \& Silverstein, M. (2014). The social network approach in gerontological research. The Journals of Gerontology: Social Sciences, 70(1), 87-90

lenca, M., Ferretti, A., Hurst, S., Puhan, M., Lovis, C., \& Vayena, E. (2018). Considerations for ethics review of big data health research: A scoping review. PloS one, 13(10), e0204937.

Lynch, C. (2008). Big data: How do your data grow?. Nature, 455(7209), 28-29.

Maddox, A. (2018). The application of digital methods in a life course approach to family studies. In, Connecting Families? Information \& Communication Technologies, generations, and the life course. Neves, B.B., and Casimiro, C. (eds). Bristol, UK: Policy Press. 97--112 
Maher, A. S., Rostowsky, K. A., Chouwdhury, N. F., \& Irimia, A. (2018, August). Neuroinformatics and Analysis of Connectomic Alterations Due to Cerebral Microhemorrhages in Geriatric Mild Neurotrauma: Microhemorrhages in Geriatric Neurotrauma. In Proceedings of the 2018 ACM International Conference on Bioinformatics, Computational Biology, and Health Informatics (pp. 165-171). ACM.

Ovadia, S. (2013). The role of big data in the social sciences. Behavioral \& Social Sciences Librarian, 32(2), 130-134.

Peine, A., \& Neven, L. (2018). From intervention to co-constitution: new directions in theorizing about aging and technology. The Gerontologist, 59(1), 15-21.

Salganik, M. J. (2017). Bit by bit: social research in the digital age. Princeton University Press.

Schulz, R., Wahl, H., Mathews, J.T., De Vito Dabbs, A., Beach, Scott R., and Czaja, Sara J. (2015). Advancing the Aging and Technology Agenda in Gerontology. The Gerontologist, 55(5), 15--21.

Singer, B. (2011). Genome-phenome linkages in human population surveys, with special emphasis on the health and retirement survey. Forum for Health Economics \& Policy. 14(3), 1--24.

Sobek, M., Cleveland, L., Flood, S., Kelly Hall, P., King, M. L., Ruggles, S., \& Schroeder, M. (2011). Big data: large-scale historical infrastructure from the Minnesota Population Center. Historical methods, 44(2), 61-68.

Weir, D. (2017). The Health and Retirement Study: Aging in the 21st Century Challenges and Opportunities for Americans. Technical report. Institute for Social Research, University of Michigan. Available online: http://hrsonline.isr.umich.edu/sitedocs/databook/?page=1 